\title{
Design and Performance Analysis of Airbag-Soft-Manipulator According to Characteristic Parameters
}

\author{
Peng Liu - Yonghong Liu* - Ke Wang - Xiaoxuan Wei - Chao Xin - Xinlei Wu \\ China University of Petroleum (East China), College of Mechanical and Electronic Engineering, China
}

\begin{abstract}
Manipulators are increasingly used in mechanical manufacturing due to the needs of industrial automation. The shortcomings of traditional manipulators, such as those in relation to their flexibility, interactivity, and safety, lead to certain limitations in their application for variable shapes and fragile objects. Given the above shortcomings of traditional manipulators, this research analysed a friction-enhanced multi-airbag soft manipulator and its performance. Analysis results of characteristic parameters show that the structure of the airbag soft manipulator is optimized, and the structural design and manufacture of the soft manipulator are completed. Deformation of the manipulator base material is described by the Neo-Hooke strain energy function, and the strain energy function coefficient is derived by obtaining the characteristic parameters of the material through tensile experiments. Finite element analysis of the manipulator is performed according to the determined strain energy function. The influence of the characteristic parameters on the mechanical bending of the manipulator is studied. Theoretical analysis proves the validity of the finite element analysis. The structural parameters of the manipulator are finally determined according to the simulation analysis results. The control system is designed according to the driving method and working mode of the soft manipulator to perform the soft manipulator grasp experiment. The manipulator can lift a weight of $300 \mathrm{~g}$, thereby indicating that the soft manipulator designed in this paper has a good application effect and prospects.
\end{abstract}

Keywords: soft manipulator, characteristic parameters, optimized, grasp experiment

\section{Highlights}

- The structure of the airbag soft manipulator is optimized, and the structural design and manufacture of the soft manipulator are completed.

- Deformation of the manipulator base material is described by the Neo-Hooke strain energy function, and the strain energy function coefficient is derived by obtaining the characteristic parameters of the material through tensile experiments.

- $\quad$ Finite element analysis of manipulator is performed according to the determined strain energy function. The influence of the characteristic parameters on the mechanical bending of the manipulator is studied.

- According to the driving method and working mode of the soft robot, the control system is designed to perform the soft robot gripping experiment.

\section{INTRODUCTION}

Robotic manipulators have reshaped industrial processes. The traditional application of rigid manipulators in people's production and life has liberated them from heavy labour, which has greatly promoted the automation and mechanization of industrial production [1]. The traditional manipulator is mainly made of metal material, which is realized by a complicated control system for handling, etc., such as the control of a six-degree-of-freedom robotic manipulator [2]. In the process of grasping irregularly shaped and fragile objects, it is difficult to perform such work using the traditional rigid manipulator because the rigidity of the operating object is much lower than the rigidity of the manipulator. With the development of three-dimensional (3D) printing technology and materials science, some scientists began to use soft materials to design and manufacture manipulators, which caused widespread interest [3] and [4]. Soft manipulators are inspired by bionics. By utilizing and exerting the mechanical intelligence of the soft material, the material is physically close to the organism itself, and the soft manipulators that are as flexible as the object to be manipulated and more adaptable are obtained [5].

To obtain mechanical properties and flexibility of the soft manipulators, many scholars have conducted a lot of research and achieved remarkable results. Marchese and Rus [6] present an extremely soft robotic manipulator morphology that is composed entirely of low durometer elastomer, powered by pressurized air, and designed to be both modular and durable. Blanes et al. [7] developed a pneumatic robot gripper capable of sorting eggplants according to their firmness, one of the fingers adapts to and copies the shapes of eggplants when the jamming of its internal granular material changes from soft to hard. Ranzani et al. [8] present the concept design of a modular soft manipulator for minimally invasive surgery, and combined flexible fluidic actuators to obtain multidirectional bending and elongation with 
a variable stiffness mechanism based on granular jamming. Erkaya [9] investigated the effects of joint clearance in a robotic system, which improved the accuracy of robotic manipulators for many industrial and medical applications. Galloway et al. [10] present the development of an underwater gripper that utilizes soft robotics technology to delicately manipulate and sample fragile species on the deep reef and demonstrated design principles for soft robot end effectors, bench-top characterization of their grasping performance and conclude by describing in situ testing at mesophotic depths.

In addition to structural improvements, many researchers have broken through the boundaries of tradition and studied the materials and manufacturing methods of software manipulators. Diller and Sitti [11] used a magnetic polymer to manufacture soft manipulators, controlling the soft manipulators by magnetic properties, so that the manipulators can achieve 3D space motion. Ge et al. [12] present a new 4D printing approach that can create high resolution (up to a few microns), multi-material shape memory polymer (SMP) architectures, and can create soft manipulators with pre-programmed functions. Yuk et al. [13] showed that the hydraulic actuations of hydrogels with designed structures and properties could give soft actuators and robots that are highspeed, high-force, and optically and sonically camouflaged in water. Ongaro et al. [14] used a thermosensitive gel to design soft untethered grippers that can be used in an unstructured environment. Hughes and Iida [15] studied the control of a generalpurpose soft manipulator based on tactile sensing.

There are also many researchers who have studied the driving methods of soft manipulators. It is hoped that the improvement of the driving method will make the soft manipulators move more quickly and more powerfully. Jun et al. [16] proposed a weighted gradient projection method to resolve inverse kinematics problems of redundant manipulators with multiple performance criteria. Brown et al. [17] developed a "coffee bag"-shaped blocking-based software manipulator that achieves the capture of objects of different shapes by blocking between different parts. Deaconescu and Deaconescu [18] aimed to present and discuss an innovative, constructive solution for a gripper system that can be attached to an industrial robot for assembly operations. Behl et al. [19] designed a soft manipulator using a shape memory polymer that can be bi-directionally bent. The soft manipulator grasps the object by bending the shape memory polymer. Kim et al. [20] developed a soft manipulator that can capture a variety of irregular objects using shape memory alloys. Lau [21] report a three-dimensional design of dielectric elastomer fingers with higher flexural stiffness and close to $90^{\circ}$ voltage-controllable bending for object gripping and pinching. Alam et al. [22] addressed the design and implementation of robust nonlinear control approaches to obtain the desired trajectory tracking of a flexible joint manipulator driven with a directcurrent geared motor. Li [23] proposed a parallel-chain nonholonomic manipulator with a chainable kinetics model. The manipulator could move to the target configuration within the specified time was proved by two simulation experiments.

Yang et al. [24] achieved both the controllability of compliance and the acquisition of position feedback in soft robotic fingers in the novel design of a 3D-printed multi-smart material substrate. Fei et al. [25] present the design and test of a novel fabric-based versatile and stiffness-tuneable soft gripper integrating soft pneumatic fingers and a wrist. The morphology is designed into a compact tuning fork shape with two bidirectional sheet-shaped soft fingers and a biaxial bidirectional (universal) cylinder-shaped soft wrist.

In this paper, an enhanced soft manipulator is designed according to the characteristics of soft materials, and the satisfying gripping effect is verified by experiments.

\section{SOFT MANIPULATOR PERFORMANCE ANALYSIS}

\subsection{Soft Manipulator Driving Method}

The function of the soft manipulator is directly affected by its structure. Such a structure includes the configuration of the soft mechanical finger and the layout of the finger. During the design process, the fingers of the soft manipulator are simulated to determine the optimal parameters. The soft manipulator is made of a generally nonlinearly deformed soft material. Thus, the conventional driving method for use on a rigid material is no longer applicable. The driving method of the software manipulator currently includes a direct transmission of the driving force from the power source to the body or driving with a directly deformable driver. The four types of commonly used driving methods include pneumatic driving [26] and [27], cable driving [28], shape memory alloy [29], and electroactive polymer driving [30]. The software manipulator studied in this research requires a substantial output force to facilitate the grasping of the object, and the soft robot must respond and be grasped quickly. Therefore, pneumatic driving is used to drive the entire soft manipulator, 
and a soft manipulator is designed according to the characteristics of the pneumatic drive.

\subsection{Neo-Hooke Characteristic Model}

A soft manipulator is distinguished from a traditional manipulator as the former uses a flexible material. Therefore, when simulating and analysing the soft manipulator, the material parameters must first be determined. In this work, silica gel is used as the base material of the manipulator. The strain energy function is often utilized to express the characteristics of the super-elastic materials of silicone rubber. Common characteristic models include the Neo-Hooke [31], Mooney-Rivlin [32], and Ogden models [33]. The most extensive model is the Neo-Hooke model, which is based on two assumptions: material incompressibility and material isotropy. Given the above assumptions, the strain energy function is described as a function of the three main invariants $I_{1}, I_{2}$ and $I_{3}$ of the Green strain tensor $E$, as shown in Eq. (1).

$$
E=E\left(I_{1}, I_{2}, I_{3}\right),
$$

where $I_{1}, I_{2}$ and $I_{3}$ can be calculated according to Eq. (2).

$$
\left\{\begin{array}{l}
I_{1}=\lambda_{1}^{2}+\lambda_{2}^{2}+\lambda_{3}^{2} \\
I_{2}=\lambda_{1}^{2} \cdot \lambda_{2}^{2}+\lambda_{1}^{2} \cdot \lambda_{3}^{2}+\lambda_{2}^{2} \cdot \lambda_{3}^{2} \\
I_{3}=\lambda_{1}^{2} \cdot \lambda_{2}^{2} \cdot \lambda_{3}^{2} \\
\lambda_{i}=1+\varepsilon_{i}
\end{array}\right.
$$

Given that the material is incompressible, Eq. (3) can be obtained as:

$$
I_{3}=\lambda_{1}^{2} \cdot \lambda_{2}^{2} \cdot \lambda_{3}^{2}=1
$$

Bringing Eq. (3) into Eq. (2) gives:

$$
\left\{\begin{array}{l}
I_{1}=\lambda_{1}^{2}+\lambda_{2}^{2}+\lambda_{3}^{2} \\
I_{2}=\frac{1}{\lambda_{1}^{2}}+\frac{1}{\lambda_{2}^{2}}+\frac{1}{\lambda_{3}^{2}}
\end{array}\right.
$$

Therefore, the strain energy function $W$ is expressed as a function of $I_{1}$ and $I_{2}$. When the material is not deformed, $\lambda_{1}=\lambda_{2}=\lambda_{3}=1$. In this condition, the strain energy function can be approximated as an exponential form, as shown in Eq. (5):

$$
W=\sum_{i+j+k=1}^{\infty} C_{i j}\left(I_{1}-3\right)^{i}\left(I_{2}-3\right)^{j}\left(I_{3}-1\right)^{k} .
$$

Given that $I_{3}=1$, the simplification of Eq. (5) is:

$$
W=\sum_{i+j=1}^{\infty} C_{i j}\left(I_{1}-3\right)^{i}\left(I_{2}-3\right)^{j} .
$$

As the limits of $I_{1}$ and $I_{2}$ are both 3, a large exponential in Eq. (6) reduces the value of $W$; thus, $W$ can be ignored in subsequent calculations. Taking the first term of Eq. (6) yields a strain energy expression in the form of a silica gel Neo-Hooke model:

$$
W=C_{10}\left(I_{1}-3\right) \text {. }
$$

Accurately describing the strain energy function of silica gel requires adding the corresponding isosceles function part to Eq. (7), and the strain energy function is obtained as follows:

$$
W=C_{10}\left(I_{1}-3\right)+D_{1}(J-1)^{2} .
$$

Simulation describes the strain of silica gel according to the strain energy function, and the coefficients therein have the following relationship:

$$
\begin{aligned}
C_{10} & =\frac{G}{2}, \\
D_{1} & =\frac{2}{\kappa} .
\end{aligned}
$$

Assuming that the volume of the silica material is incompressible, its bulk modulus is infinite, $D_{1}=0$, and the shear modulus of the material can be experimentally determined.

\subsection{Soft Material Characteristic Parameters}

To determine the material characteristic parameters, the tensile test is carried out, and the specimen is prepared according to the corresponding standardGB/T 528-2009. Fig. 1a illustrates the shape of the specimen, and Fig. $1 \mathrm{~b}$ demonstrates the tensile test specimen. The dimensions of the specimen are as follows: A (100 mm), B (25 mm), C (20 mm), D (5 $\mathrm{mm}), \mathrm{E}(11 \mathrm{~mm})$, and $\mathrm{F}(25 \mathrm{~mm})$.

A tensile test is performed. As shown in Fig. 2, the model of the tensile testing machine is the JJTEST-UTM. The specimen is symmetrically clamped on the upper and lower holders of the tensile testing machine, thereby enabling an even distribution of the tensile force on the cross-section. Fig. 2a presents the testing machine. Fig. $2 b$ illustrates the specimen clamping diagram. During the test, the tensile machine stretched the specimen at a speed of $5 \mathrm{~mm} / \mathrm{min}$ until it broke. The maximum stress is generated at the fracture of the specimen. The displacement of the chuck is automatically recorded by a tensile machine during the stretching process. The change in the length $\Delta L$ and the load generated by the stretching $F$ is obtained; then, the strain $\varepsilon$ and the stress $\sigma$ are calculated as in Eqs. (11) and (12), respectively. 
(a)


Fig. 1. Specimen; a) diagram of dumbbell specimen; and b) tensile test specimen
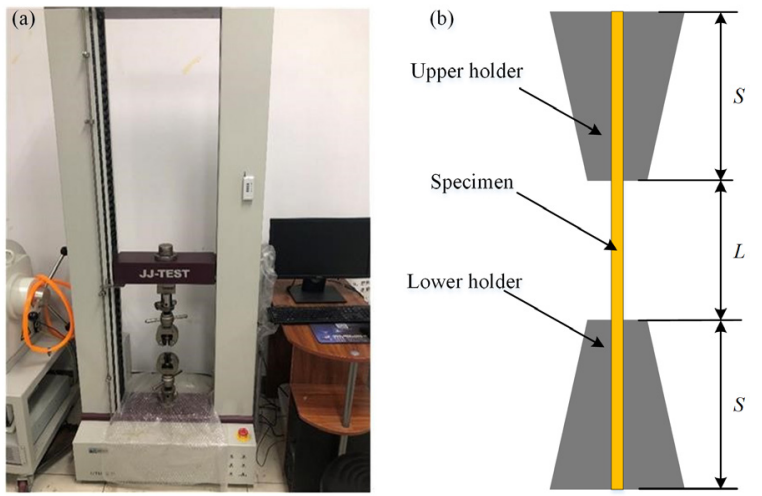

Fig. 2. Universal testing machine and its holder; a) tensile testing machine; and b) clamping diagram

$$
\begin{gathered}
\varepsilon=\frac{\Delta L}{L}, \\
\sigma=\frac{F}{S_{b}},
\end{gathered}
$$

where $L$ represents the length of the specimen before deformation, and $S_{b}$ is the cross-sectional area at the fracture. The stress-strain curve of the specimen is obtained. As shown in Fig. 3, Young's modulus of the silica material can be obtained:

$$
E=\frac{\sigma}{\varepsilon}
$$

Eq. (13) indicates that Young's modulus is the slope of the relationship curve in Fig. 3. Young's modulus of the silica gel material is obtained, namely, $E=0.67441 \mathrm{MPa}$. The following relationship exists between the shear modulus and Young's modulus:

$$
G=\frac{E}{2(1+\mu)},
$$

where $G$ is the shear modulus of the material in MPa; and $\mu$ is 0.48 .

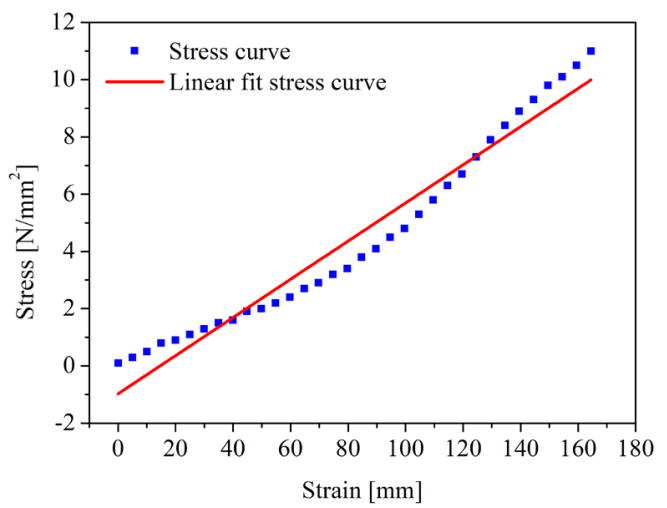

Fig. 3. Stress-strain curve of silica gel

The shear modulus of the material can be calculated through Eq. (14). According to Eq. (9), the parameters $C_{10}=0.11392$ and $D_{1}=0$ of the material under the Neo-Hooke strain energy function can be obtained.

\section{SIMULATION ANALYSIS OF THE SOFT MANIPULATOR}

\subsection{Finite Element Simulation Analysis}

After selecting gas power as the driving method of the soft manipulator, we design a multi-airbag soft manipulator. Fig. 4 shows the structure.

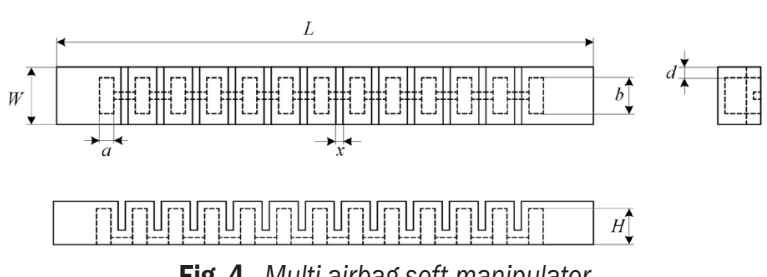

Fig. 4. Multi-airbag soft manipulator

Multi-airbag soft manipulator is affected by body characteristic parameters when subjected to force deformation, including: width $W$, length $L$, airbag width $a$, airbag length $b$, airbag depth $H$, airbag number $n$, airbag wall thickness $d$ and airbag spacing $x$. The simulation analysis initialization parameters are set to $150 \mathrm{~mm}$ (length), $16 \mathrm{~mm}$ (width), $12 \mathrm{~mm}$ (height), and the number of airbags (13). The size of a single airbag is $10 \mathrm{~mm}$ (length), $3 \mathrm{~mm}$ (width), and 10 $\mathrm{mm}$ (height). The spacing of the airbag is $10 \mathrm{~mm}$, with $2 \mathrm{~mm}$ in width for the back gap, $8 \mathrm{~mm}$ in height, and 
the spacing runs through the front and back. During the analysis, the pressure applied inside the soft manipulator is $0.07 \mathrm{MPa}$.

\section{(1) Effect of width $W$}

Under the condition that the airbag length is constant $b$, a relationship exists between the width of the soft manipulator and the thickness of the sidewall, as shown in Eq. (15):

$$
W=b+2 d .
$$

According to Eq. (15), the case wherein the airbag length is determined, the influence of the thickness of the soft body sidewall, and the soft body manipulator width on the mechanical bending of the soft body are essentially similar. When studying the influence of the width using SIMULIA Abaqus [34], the width of the soft manipulator is set to $14 \mathrm{~mm}, 16 \mathrm{~mm}, 18 \mathrm{~mm}, 20$ $\mathrm{mm}$, and $22 \mathrm{~mm}$, and Fig. 5 shows the angles of the mechanical arm of different widths.

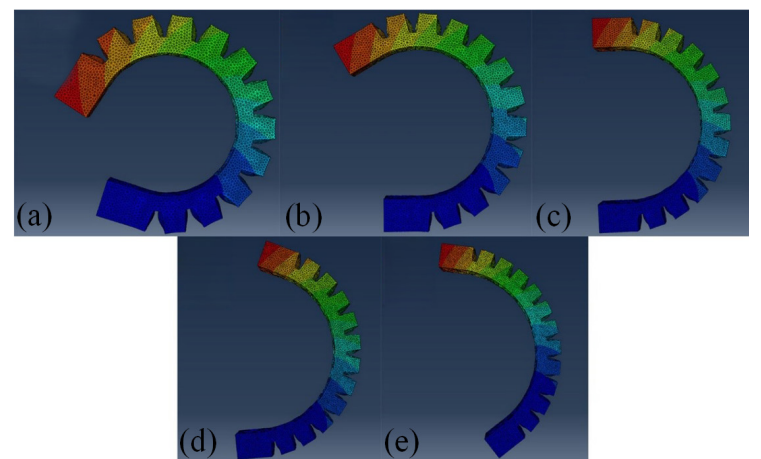

Fig. 5. Bending angle of soft manipulator with different width; a) $14 \mathrm{~mm}$; b) $16 \mathrm{~mm}$; c) $18 \mathrm{~mm}$; d) $20 \mathrm{~mm}$; and e) $22 \mathrm{~mm}$

The relationship between the width and the bending angle is ascertained, as shown in Table 1.

Table 1. Bending angle of soft manipulator under different widths

\begin{tabular}{lccccc}
\hline$W[\mathrm{~mm}]$ & 14 & 16 & 18 & 20 & 22 \\
\hline Bending angle $\left[^{\circ}\right]$ & 258.5 & 210.3 & 175.8 & 155.3 & 138.5 \\
\hline
\end{tabular}

As the width of the soft manipulator increases, the angle of the mechanical manipulator's force bending becomes smaller slowly, thereby indicating that the increase in the width of the soft manipulator negatively affects the bending of the manipulator.

(2) Effect of the airbag length $b$

When studying the effect of the width of the soft airbag on the bending of the soft manipulator, the airbag lengths are set to $8 \mathrm{~mm}, 10 \mathrm{~mm}, 12 \mathrm{~mm}$, $14 \mathrm{~mm}$, and $16 \mathrm{~mm}$. Fig. 6 illustrates the angles of the mechanical manipulators under different airbag lengths.

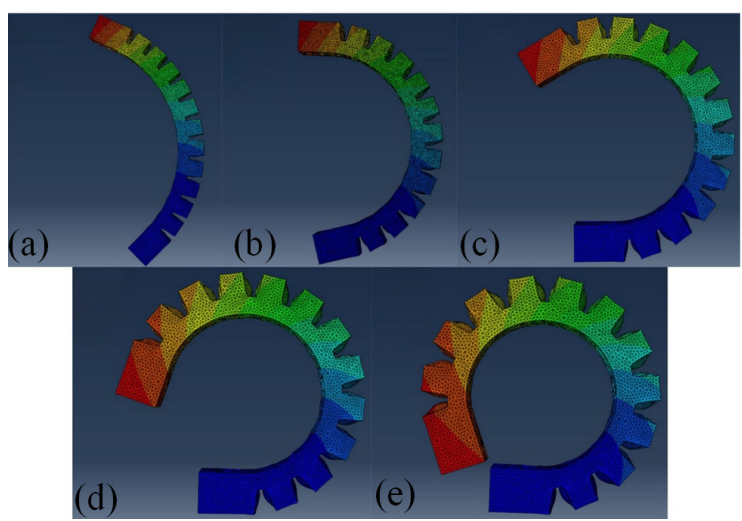

Fig. 6. Bending angle of soft manipulator under different airbag length; a) $8 \mathrm{~mm}$; b) $10 \mathrm{~mm}$; c) $12 \mathrm{~mm}$; d) $14 \mathrm{~mm}$; and e) $16 \mathrm{~mm}$

The relationship between airbag length and the bending angle is ascertained and shown in Table 2.

Table 2. Bending angle of soft manipulator under different widths

\begin{tabular}{lccccc}
\hline$b$ [mm] & 6 & 8 & 10 & 12 & 14 \\
\hline Bending angle $\left[^{\circ}\right]$ & 110.1 & 165.3 & 210.3 & 252.5 & 289.6 \\
\hline
\end{tabular}

As the length of the airbag increases, the force bending angle of the soft manipulator increases. Thus, airbag length positively influences the mechanical bending of the soft manipulator.

(3) Effect of airbag width $a$

When the airbag width a is applied to the soft manipulator, the airbag widths are set to $2 \mathrm{~mm}, 2.5$ $\mathrm{mm}, 3 \mathrm{~mm}, 3.5 \mathrm{~mm}$, and $4 \mathrm{~mm}$; moreover, Fig. 7 illustrates the bending under different airbag width conditions.

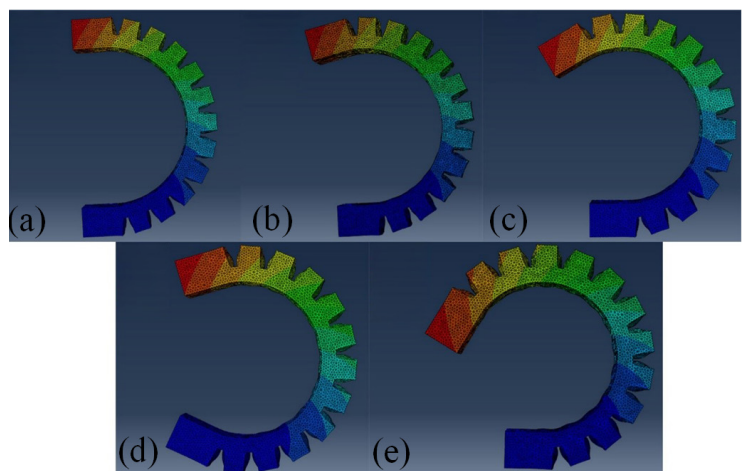

Fig. 7. Bending angle of soft manipulator under different airbag widths; a) $2 \mathrm{~mm}$; b) $2.5 \mathrm{~mm}$; c) $3 \mathrm{~mm}$; d) $3.5 \mathrm{~mm}$; and e) $4 \mathrm{~mm}$ 
Table 3 shows the relationship between the airbag width and the bending angle.

Table 3. Bending angle of soft manipulator under different airbag widths

\begin{tabular}{lccccc}
\hline$a$ [mm] & 2 & 2.5 & 3 & 3.5 & 4 \\
\hline Bending angle [ $\left.{ }^{\circ}\right]$ & 178.2 & 193.4 & 210.3 & 222.4 & 238.4 \\
\hline
\end{tabular}

As the width of the airbag increases, the angle at which the soft body is forced to bend increases, thereby indicating that the increase in the width of the airbag positively affects the bending of the soft manipulator.

(4) Effect of the number of airbags $n$

When the lengths of both ends of the soft manipulator are unchanged, the relationship between the length of the soft manipulator and the number of the airbags $n$ is expressed by Eq. (16), and the airbag pitch at this time is $10 \mathrm{~mm}$.

$$
L=d_{1}+d_{2}+x n .
$$

When the length of both ends and the distance between the airbags are constant, the length of the soft manipulator is essentially the same as the number of airbags. The number of airbags in the simulation are 11 to 15 . Fig. 8 shows the bending angle of the soft manipulator.

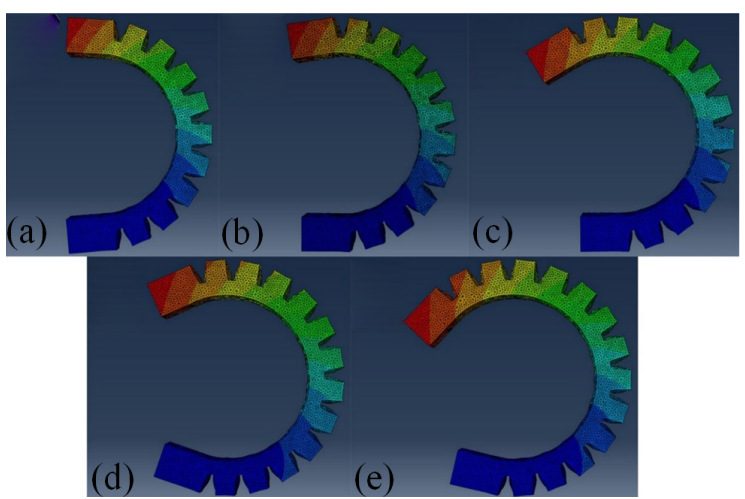

Fig. 8. Bending angle of soft manipulator under different airbag numbers; a) 11; b) 12; c) 13; d) 14; and e) 15

Table 4 shows the relationship between the number of airbags and the bending angle.

Table 4. Bending angle of soft manipulator under different airbag widths

\begin{tabular}{lccccc}
\hline$n$ & 11 & 12 & 13 & 14 & 15 \\
\hline Bending angle $\left[^{\circ}\right]$ & 171.4 & 190.0 & 210.3 & 223.6 & 240.1 \\
\hline
\end{tabular}

As the number of airbags increases, the angle at which the soft manipulator is forced to bend increases. Thus, an increase in the number of airbags positively affects the mechanical bending of the soft manipulator.

\section{(5) Effect of airbag depth $H$}

The depths of the soft body manipulator airbag are $6 \mathrm{~mm}$ to $10 \mathrm{~mm}$ in the study of the effect of the airbag depth $H$. Fig. 9 illustrates the bending under different airbag depth conditions.

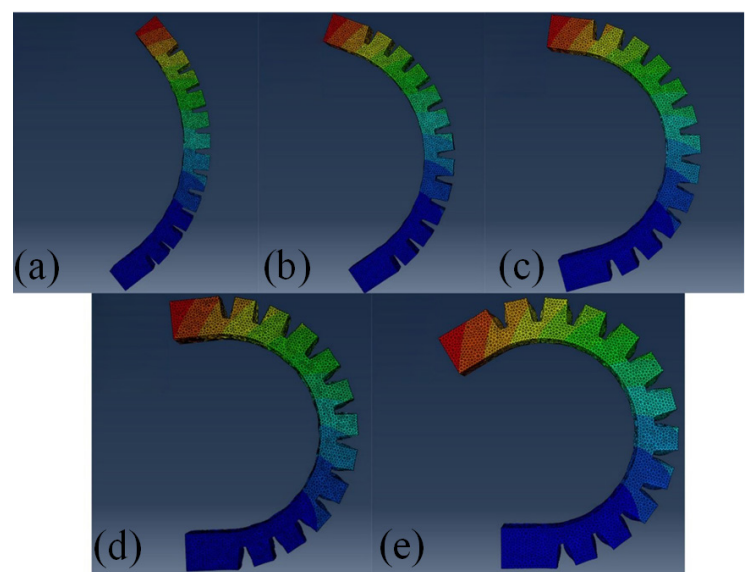

Fig. 9. Bending angle of soft manipulator under different airbag depths; a) $6 \mathrm{~mm}$; b) $7 \mathrm{~mm}$; c) $8 \mathrm{~mm}$; d) $9 \mathrm{~mm}$; and e) $10 \mathrm{~mm}$

Table 5 demonstrates the relationship between the airbag width and the bending angle.

Table 5. Bending angle of soft manipulator under different airbag depths

\begin{tabular}{lccccc}
\hline$H[\mathrm{~mm}]$ & 6 & 7 & 8 & 9 & 10 \\
\hline Bending angle $\left[^{\circ}\right]$ & 87.7 & 124.8 & 161.9 & 184.9 & 210.3 \\
\hline
\end{tabular}

As the depth of the airbag increases, the angle at which the soft body manipulator bends is also increased. Thus, an increase in the depth of the airbag positively affects the mechanical bending of the soft body manipulator.

\section{(6) Effect of airbag spacing $x$}

According to Eq. (16), the influence of the airbag spacing and of the length of the soft manipulator on the mechanical bending of the soft body are essentially the same when the length of the two ends of the soft manipulator and the number of airbags are constant. The spacing of airbags are $9 \mathrm{~mm}, 10 \mathrm{~mm}, 11$ $\mathrm{mm}, 12 \mathrm{~mm}$, and $13 \mathrm{~mm}$ in the study of the influence of the spacing of the airbag on the soft manipulator, and Fig. 10 presents the bending diagram of the soft manipulator. 


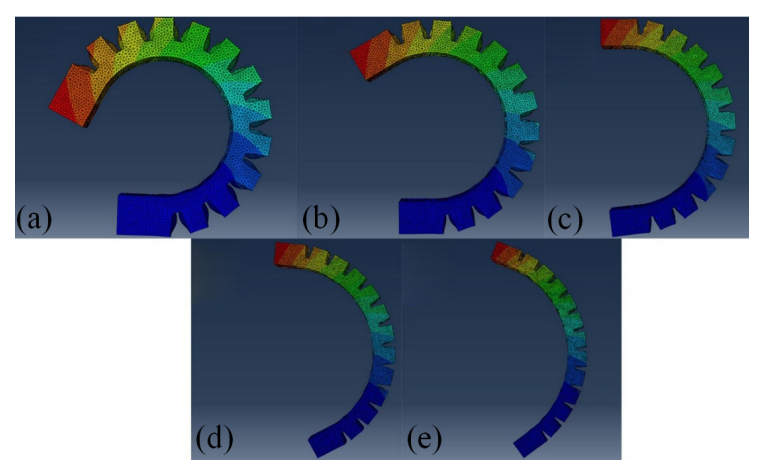

Fig. 10. Bending angle of soft manipulator under different airbag spacings; a) $9 \mathrm{~mm}$; b) $10 \mathrm{~mm}$; c) $11 \mathrm{~mm}$; d) $12 \mathrm{~mm}$; and e) $13 \mathrm{~mm}$

Table 6 establishes the relationship between the airbag spacing and the bending angle.

Table 6. Bending angle of soft manipulator under different airbag spacings

\begin{tabular}{lccccc}
\hline$x[\mathrm{~mm}]$ & 9 & 10 & 11 & 12 & 13 \\
\hline Bending angle $\left[^{\circ}\right]$ & 245.1 & 210.3 & 175.7 & 146.7 & 120.6 \\
\hline
\end{tabular}

As the distance between the airbags increases, the angle at which the soft manipulator is forced to bend is reduced. Thus, an increase in the depth of the airbag negatively affects the mechanical bending of the soft manipulator.

\subsection{Theoretical Analysis of Software Manipulator Bending}

Fig. 11 illustrates the force received inside the soft manipulator. For convenience, the force analysis inside the soft manipulator is simplified because the soft manipulator is only bent inside the illustrated interface, and the force acting on the sidewall of the soft manipulator can be ignored. Fig. 12 presents a simplified schematic of the force analysis.

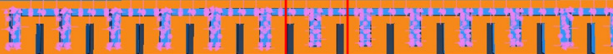

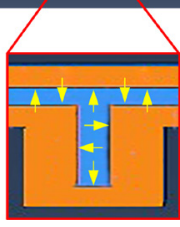

Fig. 11. Schematic diagram of the internal force of a soft manipulator

The inner wall of the soft manipulator is divided into a curved inner side and a curved outer side (Fig. 13). The inner sidewall of the curved inner side has no airbag and is a flat surface. Conversely, the inner wall

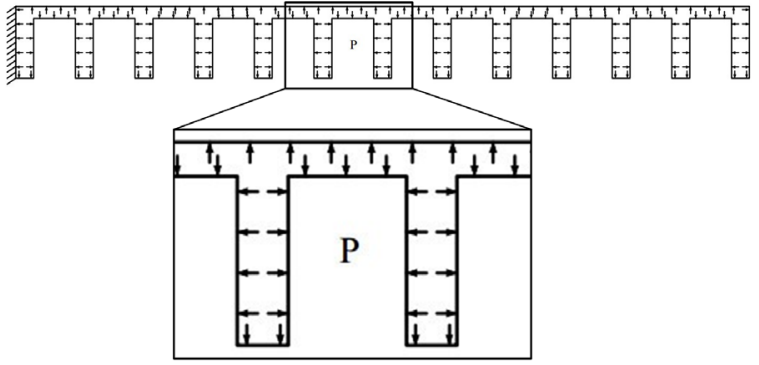

Fig. 12. Schematic diagram of force analysis for soft manipulator

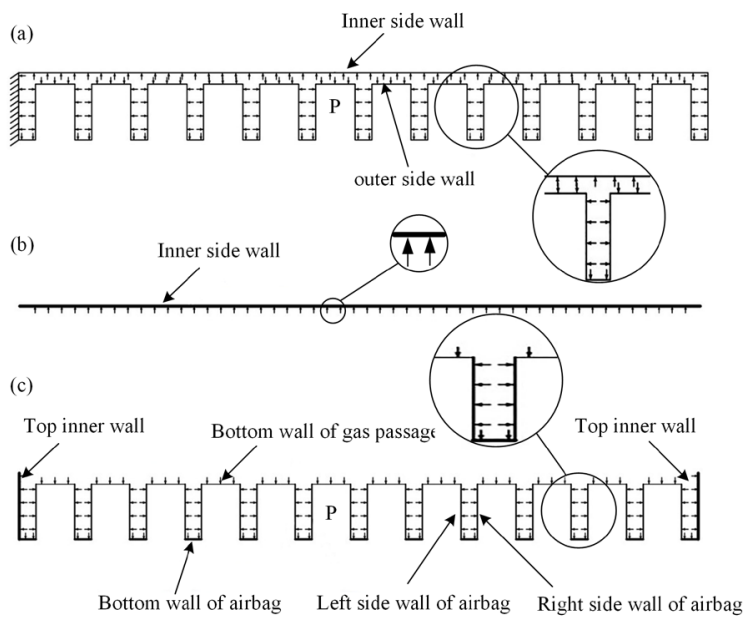

Fig. 13. Inner and outer sides of bending soft manipulators and their stress analysis; a) inner and outer sides; $b$ ) stress analysis of inner side; c) stress analysis of outer side

of the outer side of the curved side is provided with an airbag. Thus, the inner wall of the force-receiving inner wall includes the inner wall of the end portion and the left side of the airbag, apart from the portion which is balanced with the inner wall of the curved inner and right side walls of the airbag. After being subjected to the same amount of gas pressure, the inner wall of the curved outer side is subjected to a pressure substantially stronger than that applied to the inner side of the curved portion, such that the force deformation generated outside the curved portion is larger than the bending deformation generated by the inner side of the curved portion, and the soft body mechanical force is bent by the force. The deformation predisposes the airbag on the outside of the curved body to expand. After being subjected to the internal pressure of the soft manipulator, the length of the curved outer side perpendicular to the fixed end is greater than the length of the curved inner side perpendicular to the fixed end owing to the curved inner side and the curved outer side. The ends of the soft manipulator are connected to each other; thus, the 
soft manipulator can only eliminate the difference in length between the inner and outer sides of the bend by bending.

The soft manipulator forms a concentric fanshaped area after bending, and the curved inner and outer sides are respectively two arcs of a fan shape. The difference between the lengths of the curved inner and outer sides can be expressed by the difference in the arc length of the fan shape (Fig. 14). The difference in the curvature of the inner and outer sides of the curved body of the soft body is obtained, as shown in Eq. (17).

$$
L_{r}=\pi \alpha\left(r_{a}+h\right)-\pi \alpha r_{a}=\pi \alpha h .
$$

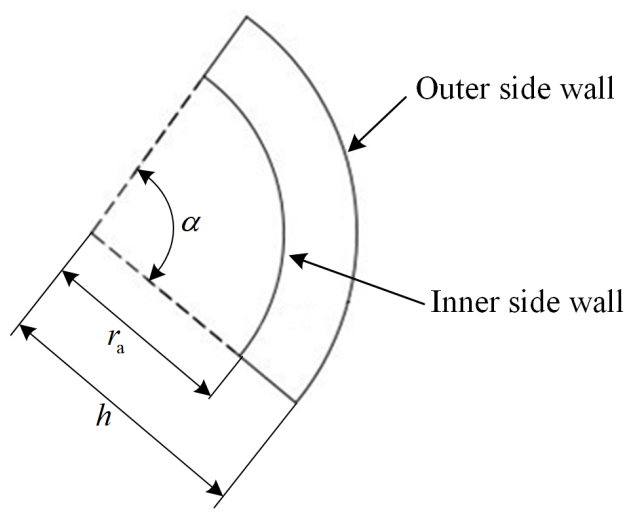

Fig. 14. A sector formed by the bending of a manipulator

The difference between its inner and outer sides is related to the height and bending curvature of the soft manipulator. After the structure of the soft manipulator is determined, the difference between the inner and outer lengths is ascertained only in relation to the arc corresponding to the curved arc. The greater the arc of the inner and outer sides, the greater the difference in arc length. The difference between the inner and outer lengths is related to the pressure difference between the inner and outer sides of the soft manipulator. The force inside the soft manipulator can be obtained by Eq. (18):

$$
F_{s}=P_{s}: S_{s}
$$

When the gas pressure applied to the inner wall of the soft manipulator is constant, the pressure applied to the inside of the soft manipulator changes with the change in the force-receiving area. The pressure difference can be obtained by Eq. (19):

$$
F_{\Delta}=P_{s} \cdot S_{\Delta},
$$

where $F_{\Delta}$ denotes the force difference between the inner and outer inner walls of the bend; $S_{\Delta}$ is the difference between the inner and outer inner wall areas of the soft manipulator.

The difference between the inner and outer inner wall areas of the soft manipulator can be calculated by Eq. (20):

$$
S_{\Delta}=S_{L}+S_{R}+S_{L T}+S_{R T} .
$$

Fig. 15 shows the force-receiving surfaces on the left and right sides of the airbag and the left and right ends of the robot.
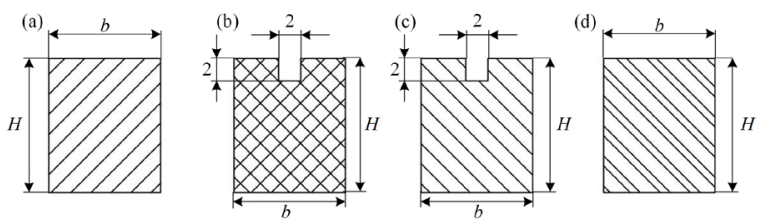

Fig. 15. Four cross-sections causing pressure difference

The four pressure differences can be calculated by Eq. (21):

$$
\left\{\begin{array}{l}
S_{L}=S_{R}=b \cdot H-4 \\
S_{L T}=S_{R T}=b \cdot H
\end{array},\right.
$$

where $b$ and $H$ respectively represent the length and depth of the soft manipulator airbag.

Eq. (21) shows that the length and depth of the airbag are strongly affected by the force of the soft manipulator. This observation can also be proven via simulation results. Under the influence of two factors, the change angles of the bending angle of the soft manipulator are $122.6^{\circ}$ and $179.5^{\circ}$, respectively. The large length influence is caused by the change amount in length, which is relatively large. The remaining influencing factors include the width $W$ and the airbag spacing $x$. The thickness of the silica gel is affected by the impedance change when the deformation is changed. The bending forces of the soft body are $-120.0^{\circ}$ and $-124.5^{\circ}$, respectively. The increase in the width of the soft manipulator and the distance between the airbags cause an increase in the bending resistance, and such modification reduces the bending angle. The width of the airbag and the number of airbags also have a certain influence on the bending of the soft manipulator. The changes in the angle are $60.2^{\circ}$ and $68.7^{\circ}$. The change in these two parameters alters the length of the soft manipulator, the width of the airbag, and the number of airbags. The increase in those parameters will increase the length of the soft manipulator; moreover, the force and deformation resistance will not change, thereby changing the bending angle of the soft manipulator. The theoretical analysis proves that the influence of the above 
parameters on the bending of the soft manipulator is consistent with the simulation analysis results, which can be used as a reference for design.

\subsection{Characteristic Parameters of the Soft Manipulator}

(1) The template size used in this research is 20 $\mathrm{mm} \times 20 \mathrm{~mm}$. For the nanofibre film and the soft manipulator to fit well, the width of the soft manipulator is set as $20 \mathrm{~mm}$.

(2) The sidewalls of the soft manipulator must have a certain thickness. When the wall thickness is under $1 \mathrm{~mm}$, the simulation results do not converge. To ensure the safety of the soft manipulator, the wall thickness must be greater than or equal to $2 \mathrm{~mm}$.

(3) According to the width and wall thickness requirements, the length of the soft manipulator airbag is $14 \mathrm{~mm}$, and the sidewall is $3 \mathrm{~mm}$, dimensions which meet the design requirements.

(4) The width of the airbag has less influence on the soft manipulator. To facilitate the draft, the width of the airbag is set to $3 \mathrm{~mm}$.

(5) The depth of the airbag is $10 \mathrm{~mm}$, where the airflow passage is distributed at $2 \mathrm{~mm}$ on the top.

(6) After the depth of the airbag is determined, the height is also ascertained to be $14 \mathrm{~mm}$, and the thickness of the upper and lower end faces is 2 $\mathrm{mm}$.

(7) To facilitate the drafting operation, the depth and width of the groove are set to $8 \mathrm{~mm}$ and 3 $\mathrm{mm}$, respectively; moreover, the thickness of the sidewall of the airbag sandwiched between the groove and the airbag is $3 \mathrm{~mm}$. These dimensions satisfy the requirements of safety design.

(8) The chosen number of airbags is eight.

(9) According to Eqs. (4) and (7) and the wall thickness of the airbag, the distance between the airbags is determined to be $12 \mathrm{~mm}$.

(10) The total length of the soft manipulator is set to $115 \mathrm{~mm}$.

(11) The two ends of the soft manipulator are symmetrical. According to Eqs. (4), (8), (9), and (10), the length of both ends is $14 \mathrm{~mm}$.

\section{GRASP EXPERIMENT OF AIRBAG SOFT MANIPULATOR}

Since the soft manipulator contains airbags inside, it cannot be integrally cast during the manufacturing process, so the moulds are separately designed and cast, and the manufactured samples are combined. The manipulator is divided into upper and lower parts, and the moulds are printed by $3 \mathrm{D}$ printing technology, as shown in Fig. 16.

The airbag soft manipulator is manufactured, as shown in Fig. 17. The driving mode of the soft manipulator is a pneumatic drive. By controlling the gas pressure inside the soft manipulator, the bending degree of the soft manipulator is directly affected; thus, the corresponding gripping force is obtained. According to the requirements of the pneumatic drive control method, the control process of the entire control system is obtained (Fig. 18).

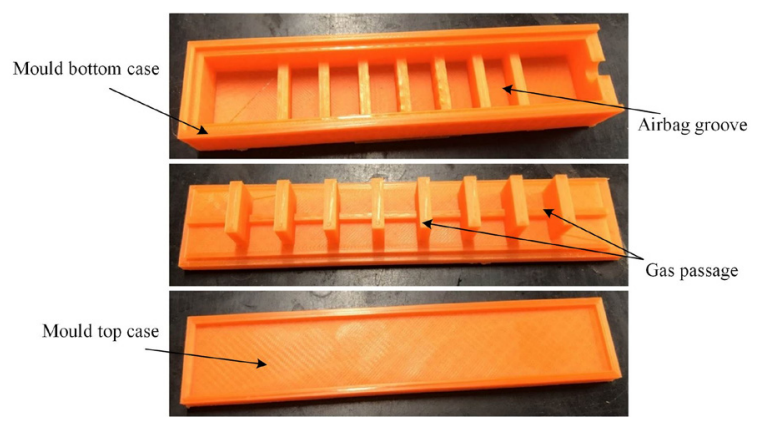

Fig. 16. Mould of soft manipulator
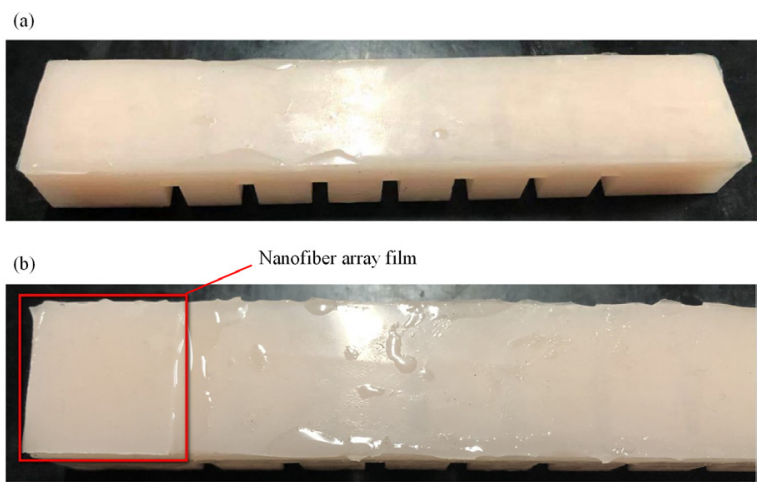

Fig. 17. Soft manipulator; a) manipulator with no nanofibre array film attached; and b) manipulator with nanofibre array film attached

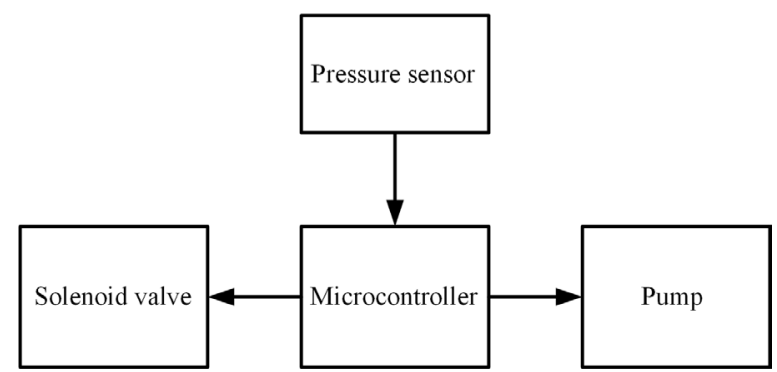

Fig. 18. Soft manipulator internal gas control process

In current robotics applications, the urgent need for productivity and precision highlights the crucial need to replace traditional control strategies 
with modern ones. Therefore, closed-loop feedback control is necessary [35] to [37]. In this paper, the control system is divided into four main parts: a microcontroller that plays a major control role (Arduino Mega 2560), a pump (DC miniature diaphragm pump) that supplies gas pressure, a valve that controls the internal communication between the pump (SMC-VQ110U-5M model solenoid valve) and the soft manipulator, and a pressure sensor that measures the gas pressure inside the manipulator in real-time. The microcontroller is the core control component that reads the pressure value transmitted by the pressure sensor and controls the opening and closing of the pump switches and valves to control the flow of air to the soft manipulator. According to the control requirements, the control strategies are set as shown in Fig. 19.

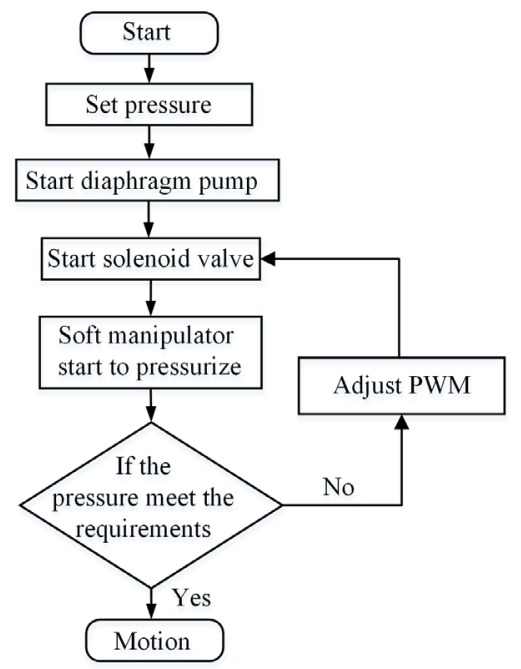

Fig. 19. Control strategy of soft manipulator

Experimental steps:

(1) Adjust the potentiometer and set the solenoid valve gas pressure.

(2) Open the diaphragm pump so that it begins to provide pressure to the system.

(3) Open the solenoid valve to allow gas to pass inside the software robot.

(4) The pressure sensor that is electrically connected to the soft manipulator measures the gas pressure inside in real-time and transmits the measured pressure data to the microcontroller.

(5) If the pressure meets the requirements, then, no adjustment is made; if the pressure fails to meet the expected value, then, the potentiometer is adjusted to modify the duty cycle of the pulse width modulation (PWM) signal, thereby controlling the frequency of the opening and closing of the solenoid valve and the gas inside the soft manipulator.

Fig. 20 shows the grasp experiment system of the soft manipulator. The pressure test is performed on each pressure channel, and the pressure value of the sensor is obtained, as shown in Fig. 21.

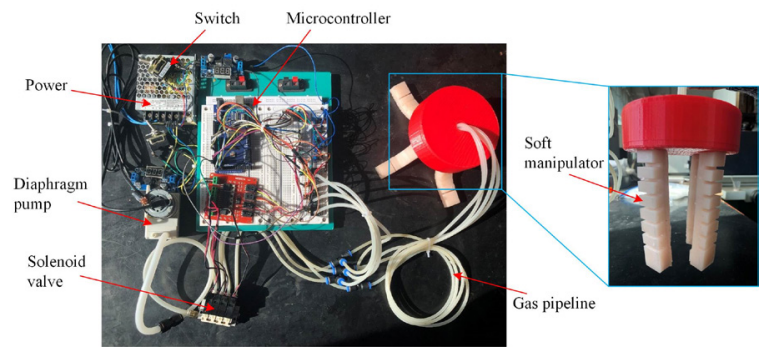

Fig. 20. Grasp experiment system of soft manipulator

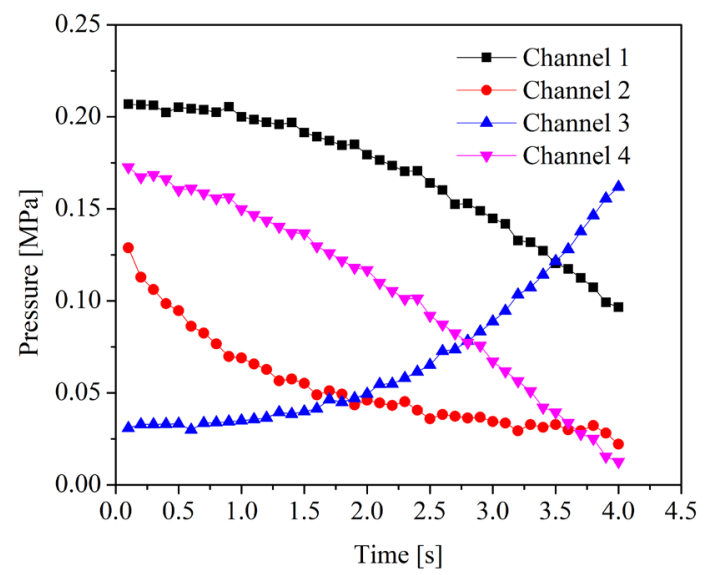

Fig. 21. Variation of pressure in each channel during testing

The pressure changes can be seen in Fig. 21 . The pressure of the four channels partially changes when the potentiometer is adjusted, thereby indicating that the system can work normally. Moreover, the pressure of the four-channel system exceeds0.1 MPa. According to the simulation results, this pressure can fully meet the requirements of the soft manipulator bending.

The stress test of a single manipulator are performed, and its bending angles under different pressures are tested. Subsequently, the test and simulation results are compared (Fig. 22). The testing pressures are set as $0.1 \mathrm{MPa}, 0.2 \mathrm{MPa}, 0.3 \mathrm{MPa}, 0.4$ $\mathrm{MPa}$, and $0.5 \mathrm{MPa}$.

The bending angles under the finite element analysis and under the pressure test fit well (Fig. 22), thereby proving that the simulation results of the soft manipulator are reliable and can be used as the basis for the design of the soft manipulator structure. 


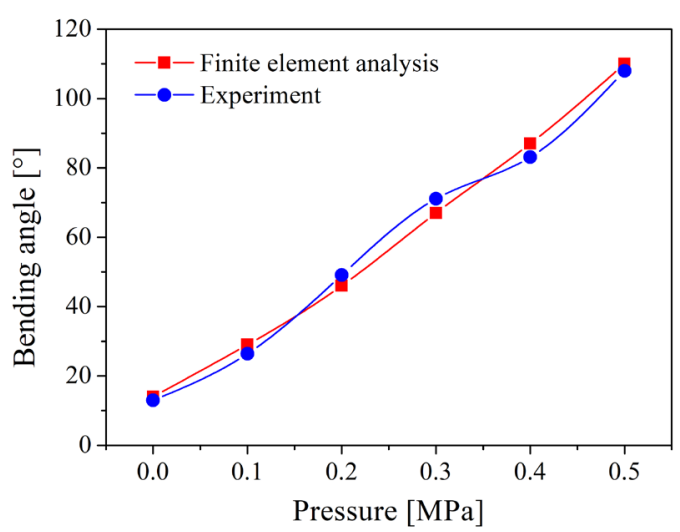

Fig. 22. Bending angle of soft manipulator under finite element analysis and experimental analysis

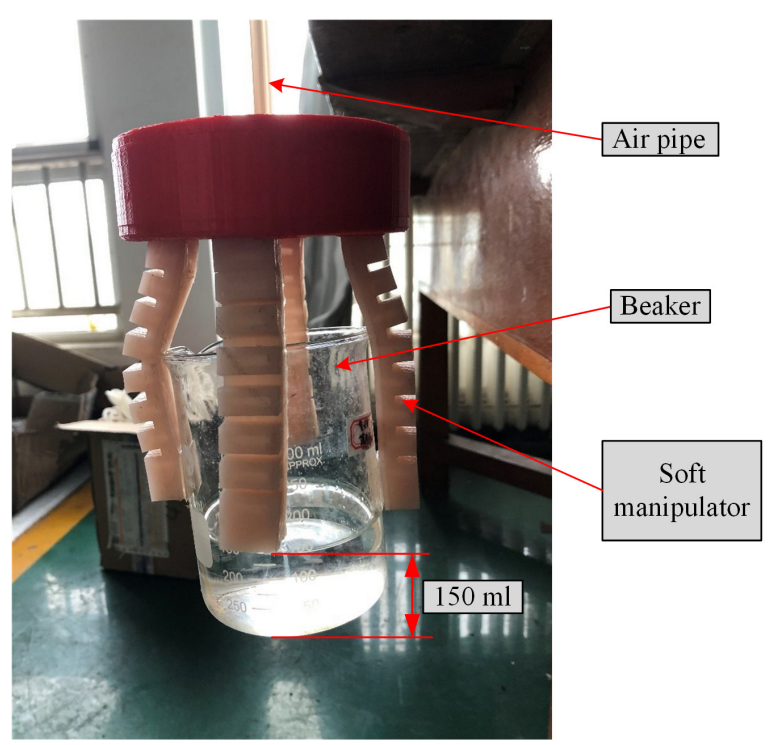

Fig. 23. Grasp experiment of soft manipulator

The grasp experiment is carried out using the built-up soft manipulator measurement and control system. The object to be grabbed is a beaker. The original weight of the beaker is $147.7 \mathrm{~g}$. Water is added to the beaker to increase the weight of the grabbed object, and Fig. 23 illustrates the grasping process. In the experiment, the final water addition is approximately $150 \mathrm{ml}$; that is, the final grab weight of the soft manipulator is $300 \mathrm{~g}$ and indicates that the soft manipulator optimization has good application effect.

\section{CONCLUSION}

A friction-enhanced multi-airbag soft manipulator is developed in the paper, and the pneumatic drive is selected as the driving method of the software robot. Firstly, finite element analysis of the manipulator is performed. Under the influence of the length and depth, the change angles of the bending angle of the soft manipulator are $122.6^{\circ}$ and $179.5^{\circ}$, respectively. The remaining influencing factors include the width $\mathrm{W}$ and the airbag spacing $x$. The thickness of the silica gel is affected by the impedance change when the deformation is changed. The bending forces of the soft body are $-120.0^{\circ}$ and $-124.5^{\circ}$, respectively. The width of the airbag and the number of airbags also have a certain influence on the bending of the soft manipulator. The changes in the angle are $60.2^{\circ}$ and $68.7^{\circ}$. The change in these two parameters alters the length of the soft manipulator, the width of the airbag, and the number of airbags. The increase in those parameters will increase the length of the soft manipulator; moreover, the force and deformation resistance will not change, thereby changing the bending angle of the soft manipulator. Secondly, the expression of the Neo-Hooke strain energy function is obtained, according to the mechanical tensile test. According to the strain energy function, the finite element analysis method is utilized to examine the influence of the change in soft manipulator size parameters on the force bending. The theoretical analysis results confirm that the finite element analysis outcomes are correct. Finally, the software manipulator is manufactured and the pressure channel tests, bending angle tests, and grasp experiment are carried out. The weight of $300 \mathrm{~g}$ can be lifted by the manipulator, thereby indicating that the soft manipulator designed in this research has good application effect and prospect.

\section{ACKNOWLEDGEMENTS}

The authors wish to acknowledge the National Natural Science Foundation of China (No. 51779267), financial support of Open Fund of Key Pre-Research Foundation of Military Equipment of China (No.6140923030702), Doctoral Innovation Engineering Project (No. YCX2019056).

\section{NOMENCLATURES}

$\alpha \quad$ Arc corresponding to the bending, $\left[{ }^{\circ}\right]$

$\lambda_{i} \quad$ Main elongation ratio

$\varepsilon_{i} \quad$ Main direction strain, [MPa]

$\kappa \quad$ Bulk modulus of the material

$\mu \quad$ Poisson's ratio of the material

$r_{a}$ The radius of the arc, [mm]

$h$ Soft manipulator height, [mm]

$G$ Shear modulus of the material, [MPa]

$F_{\Delta}$ Inner and outer wall force difference, [N]

$F_{s}$ Force on the inner wall, [N] 
$L_{T} \quad$ Inner and outer arc length difference, $[\mathrm{mm}]$

$P_{S} \quad$ Gas pressure received by the inner wall, [MPa]

$S_{\Delta} \quad$ Inner and outer wall area difference, $\left[\mathrm{mm}^{2}\right]$

$S_{L} \quad$ Left side of the airbag, $\left[\mathrm{mm}^{2}\right]$

$S_{L T}$ Left end area of the manipulator, $\left[\mathrm{mm}^{2}\right]$

$S_{R} \quad$ Right side of the airbag, $\left[\mathrm{mm}^{2}\right]$

$S_{R T}$ Right end area of the manipulator, $\left[\mathrm{mm}^{2}\right]$

$S_{s} \quad$ Area of force inside the soft manipulator, $\left[\mathrm{mm}^{2}\right]$

$W$ Strain energy function

\section{REFERENCES}

[1] Iqbal, J., Ullah, M.I., Khan, A.A., Irfan, M. (2015). Towards sophisticated control of robotic manipulators: An experimental study on a pseudo-industrial arm. Strojniški vestnik - Journal of Mechanical Engineering, vol. 61, no. 7-8, p. 465-470, D0l:10.5545/sv-jme.2015.2511.

[2] Ajwad, S.A., Iqbal, J., Islam, R.U., Alsheikhy, A., Almeshal, A., Mehmood, A. (2018). Optimal and robust control of multi DOF robotic manipulator: Design and hardware realization. Cybernetics and Systems, vol. 49, no.1, p. 77-93, Dol:10.1080 /01969722.2017.1412905.

[3] Rus, D., Tolley, M.T. (2015). Design, fabrication and control of soft robots. Nature, vol. 521, no. 7553 , p. 467-475, DOl:10.1038/nature14543.

[4] Elango, N., Faudzi, A.A.M. (2015). A review article: investigations on soft materials for soft robot manipulations. The International Journal of Advanced Manufacturing Technology, vol. 80, no. 5-8, p. 1027-1037, Dol:10.1007/ s00170-015-7085-3.

[5] Dupac, M., Noroozi, S. (2014). Dynamic modeling and simulation of a rotating single link flexible robotic manipulator subject to quick stops. Strojniški vestnik - Journal of Mechanical Engineering, vol. 60, no. 7-8, p. 475-482, D0I:10.5545/sv-jme.2013.1544.

[6] Marchese, A.D., Rus, D. (2016). Design, kinematics, and control of a soft spatial fluidic elastomer manipulator. The International Journal of Robotics Research, vol. 35, no. 7, p. 840-869, DOl:10.1177/0278364915587925.

[7] Blanes, C., Ortiz, C., Mellado, M., Beltrán, P. (2015). Assessment of eggplant firmness with accelerometers on a pneumatic robot gripper. Computers and Electronics in Agriculture, vol. 113, p. 44-50, D0l:10.1016/j. compag.2015.01.013.

[8] Ranzani, T., Cianchetti, M., Gerboni, G., De Falco, I., Menciassi, A. (2016). A soft modular manipulator for minimally invasive surgery: design and characterization of a single module. IEEE Transactions on Robotics, vol. 32, no. 1, p. 187-200, DOI:10.1109/TRO.2015.2507160.

[9] Erkaya, S. (2018). Effects of joint clearance on the motion accuracy of robotic manipulators. Strojniški vestnik Journal of Mechanical Engineering, vol. 64, no. 2. p. 82-94, D0I:10.5545/sv-jme.2017.4534.

[10] Galloway, K.C., Becker, K.P., Phillips, B., Kirby, J., Licht, S., Tchernov, D., Wood, R.J., Gruber, D.F. (2016). Soft robotic grippers for biological sampling on deep reefs. Soft Robotics, vol. 3, no. 1, p. 23-33, D0l:10.1089/soro.2015.0019.
[11] Diller, E., Sitti, M. (2014). Three-dimensional programmable assembly by untethered magnetic robotic micro-grippers. Advanced Functional Materials, vol. 24, no. 28, p. 4397-4404, DOl:10.1002/adfm.201400275.

[12] Ge, Q., Sakhaei, A.H., Lee, H., Dunn, C.K., Fang, N.X., Dunn, M.L. (2016). Multimaterial 4D printing with tailorable shape memory polymers. Scientific Reports, vol. 6, p. 31110, DOl:10.1038/srep31110.

[13] Yuk, H., Lin, S., Ma, C., Takaffoli, M., Fang, N.X., Zhao, X. (2017). Hydraulic hydrogel actuators and robots optically and sonically camouflaged in water. Nature Communications, vol. 8, p. 14230, DOI:10.1038/ncomms14230.

[14] Ongaro, F., Scheggi, S., Yoon, C., van den Brink, F., Oh, S.H., Gracias, D.H., Misra, S. (2017). Autonomous planning and control of soft untethered grippers in unstructured environments. Journal of Micro-Bio Robotics, vol. 12, no. 1-4, p. 45-52, DOl:10.1007/s12213-016-0091-1.

[15] Hughes, J., lida, F. (2018). Tactile sensing applied to the universal gripper using conductive thermoplastic elastomer. Soft Robotics, vol. 5, no. 5, p. 512-526, D0l:10.1089/ soro.2017.0089.

[16] Wan, J., Yao, J., Zhang, L., Wu, H. (2018). A weighted gradient projection method for inverse kinematics of redundant manipulators considering multiple performance criteria. Strojniški vestnik - Journal of Mechanical Engineering, vol. 64, no. 7-8. p. 475-487, D0l:10.5545/sv-jme.2017.5182.

[17] Brown, E., Rodenberg, N., Amend, J., Mozeika, A., Steltz, E., Zakin, M.R., Lipson, H., Jaeger, H.M. (2010). Universal robotic gripper based on the jamming of granular material. Proceedings of the National Academy of Sciences, vol. 107, no. 44, p. 18809-18814, D0l:10.1073/pnas.1003250107.

[18] Deaconescu, T., Deaconescu, A. (2017). Pneumatic muscleactuated adjustable compliant gripper system for assembly operations. Strojniški vestnik - Journal of Mechanical Engineering, vol. 63 , no. 4, p. 225-235, Dol:10.5545/svjme.2016.4239.

[19] Behl, M., Kratz, K., Zotzmann, J., Nöchel, U., Lendlein, A. (2013). Reversible bidirectional shape-memory polymers. Advanced Materials, vol. 25, no. 32, p. 4466-4469, DOl:10.1002/adma.201300880.

[20] Kim, H.-I., Han, M.-W., Wang, W., Song, S.-H., Rodrigue, H., Ahn, S.-H. (2015). Design and development of bio-mimetic soft robotic hand with shape memory alloy. 2015 IEEE International Conference on Robotics and Biomimetics, $p$. 2330-2334, DOI:10.1109/ROBI0.2015.7419122.

[21] Lau, G.-K., Heng, K.-R., Ahmed, A.S., Shrestha, M. (2017). Dielectric elastomer fingers for versatile grasping and nimble pinching. Applied Physics Letters, vol. 110, no. 18, p. 182906, DOl:10.1063/1.4983036.

[22] Alam, W., Mehmood, A., Ali, K., Javaid, U., Alharbi, S., Iqbal, J. (2018). Nonlinear control of a flexible joint robotic manipulator with experimental validation. Strojniški vestnik Journal of Mechanical Engineering, vol. 64, no. 1, p. 47-55, DOI:10.5545/sv-jme.2017.4786.

[23] Li, L. (2018). Mechanism design and motion planning of parallel-chain nonholonomic manipulator. International Journal of Simulation Modelling, vol. 17, no. 2, p. 327-336, DOI:10.2507/IJSIMM17(2)C07. 
[24] Yang, Y., Chen, Y., Li, Y., Wang, Z., Li, Y. (2017). Novel variablestiffness robotic fingers with built-in position feedback. Soft Robotics, vol. 4, no. 4, p. 338-352, D0l:10.1089/ soro.2016.0060.

[25] Fei, Y., Wang, J., Pang, W. (2019). A novel fabric-based versatile and stiffness-tunable soft gripper integrating soft pneumatic fingers and wrist. Soft Robotics, vol. 6, no. 1, p. 1-20, DOI:10.1089/soro.2018.0015.

[26] Connolly, F., Polygerinos, P., Walsh, C.J., Bertoldi, K. (2015). Mechanical programming of soft actuators by varying fiber angle. Soft Robotics, vol. 2, no. 1, p. 26-32, Dol:10.1089/ soro.2015.0001.

[27] Jang, J.-S. (2005). Simultaneous trajectory tracking control of position and force with pneumatic cylinder driving apparatus. Journal of Mechanical Science and Technology, vol. 19, no. 5, p. 1107-1115, DOI:10.1007/BF02984032.

[28] Dong, H., Asadi, E., Qiu, C., Dai, J., Chen, I.-M. (2018). Geometric design optimization of an under-actuated tendon-driven robotic gripper. Robotics and ComputerIntegrated Manufacturing, vol. 50, p. 80-89, D0l:10.1016/j. rcim.2017.09.012.

[29] Rodrigue, H., Bhandari, B., Han, M.-W., Ahn, S.-H. (2015). A shape memory alloy-based soft morphing actuator capable of pure twisting motion. Journal of Intelligent Material Systems and Structures, vol. 26, no. 9, p. 1071-1078, DOI:10.1177/1045389X14536008.

[30] O'Halloran, A., O'Malley, F., McHugh, P. (2008). A review on dielectric elastomer actuators, technology, applications, and challenges. Journal of Applied Physics, vol. 104, no. 7, p. 9, DOI:10.1063/1.2981642.

[31] Treloar, L.R.G. (1944). Stress-strain data for vulcanized rubber under various types of deformation. Rubber Chemistry and Technology, vol. 17, no. 4, p. 813-825, Dol:10.5254/1.3546701.

[32] Mooney, M.A. (1940). A theory of large elastic deformation. Journal of Applied Physics, vol. 11, no. 9, p. 582-592, DOI:10.1063/1.1712836.

[33] Ogden, R.W. (1982). Elastic deformations of rubberlike solids. Mechanics of Solids, p. 499-537, D0I:10.1016/B978-0-08025443-2.50021-5.

[34] Ramadani, R., Belsak, A., Kegl, M., Predan, J., Pehan, S. (2018). Topology optimization based design of lightweight and low vibration gear bodies. International Journal of Simulation Modelling, vol. 17, no. 1, p. 92-104, D0l:10.2507/ ijsimm17(1)419.

[35] Li, Y., Liu, L., Feng, G. (2018). Robust adaptive output feedback control to a class of non-triangular stochastic nonlinear systems. Automatica, vol. 89, p. 325-332, D0I:10.1016/j. automatica.2017.12.020.

[36] Ajwad, S.A., Iqbal, J., Ullah, M.l., Mehmood, A. (2015). A systematic review of current and emergent manipulator control approaches. Frontiers of Mechanical Engineering, vol. 10, no. 2, p. 198-210, Dol:10.1007/s11465-015-0335-0.

[37] Vidaković, J., Kvrgić, V., Lazarević, M. (2018). Control system design for a centrifuge motion simulator based on a dynamic model. Strojniški vestnik - Journal of Mechanical Engineering, vol. 64, no. 7-8, p. 465-474, D0l:10.5545/sv-jme.2018.5272. 